\title{
LA RÉTENTION MNÉMONIQUE CHEZ APIS MELLIFICA
}

PAR

\section{Maurice VUILLAUME.}

Station de Recherches apicoles, Bures/Yvette.

De nombretx cas de mémoire, liés plus ou moins à l'apprentissage, ont été signalés ces dernières années chez les insectes. Mais la durée de leur rétention mnémonique est en général assez courte. Dans le dressage au labyrinthe, les Blattes ne paraissent pas retenir plus d'une demi-heure le chemin à suivre (Blatella, Chauvin) ou plus de quelques heures (Periplaneta, BrECHER). Les Dytiques peuvent au contraire, conserver plusieurs jours l'habitude d'associer la présence de nourriture à une saveur donnée (SCHALLLER). L Les abeilles dressées à venir chercher de la nourriture sur un panneau coloré s'intéressent encore à lui quatre jours après (K. Von FrIsch) ; les papillons, dans les mêmes conditions oublient tout dès le lendemain (Von FrISch, I9I4) : mais ils retiennent beaucoup mieux les odeurs et pourraient même s'en servir après plusieurs semaines pour leur orientation (Von FRISCH, I920).

Je voudrais faire ici quelques remarques sur la rétention mnémonique chez l'abeille, qui paraît beaucoup plus étendue que ce qu'en disent les auteurs; et l'on peut se demander si cette rétention ne peut varier considérablement suivant les circonstances de la vie et les conditions de l'observation.

\section{LA MÉMOIRE DU RETOUR CHEZ L'ABEILLE}

Je me contenterai ici de quelques remarques, le sujet ayant été abondamment traité, notamment dans une discussion récente de Von Frisch et Lindauer (I956). On sait que la mémoire précise de la topographie et de l'emplacement de la ruche permet aux abeilles de retrouver leur colonie après un vol qui les en a éloignées de plusieurs kilomètres (jusqu'à II km). Si par contre la ruche elle-même est éloignée artificielle- 
ment de plus de $3 \mathrm{~km}$ par rapport à l'emplacement initial, les abeilles s'orientent par rapport au nouvel emplacement de la ruche et ne reviennent pas à l'ancien ; dans ce cas, la présence de la reine peut jouer un rôle fixateur important; mais elle ne suffit pas à elle seule à maintenir les abeilles en place. Il suffit en effet de déplacer la ruche $\grave{a}$ moins de $3 \mathrm{~km}$ pour que toutes les butineuses retournent à leur ancien emplacement.

Ceci nous amène à distinguer plusieurs zones autour de la ruche : 0 à $3 \mathrm{~km}-3$ à II $\mathrm{km}$ - o à $\mathrm{rI} \mathrm{km}$.

a) les abeilles d'une portion orpheline de la colonie prélevée et mise en ruche dans la zone $3^{-I I} \mathrm{~km}$ ne retournent pas à leur ancien emplacement.

b) déplacées dans la zone $0-3 \mathrm{~km}$ avec ou sans reine, les butineuses retournent à l'emplacement initial ;

c) dans la zone o-II $\mathrm{km}$, les abeilles sont capables, en partant de la ruche, de retrouver son emplacement.

Ces données que tous les apiculteurs utilisent journellement prouvent qu'une abeille connaît et garde en sa mémoire la topographie de la zone située autour de la ruche, zone inscrite dans un cercle de $3 \mathrm{~km}$ de rayon dont la colonie forme le centre. Elles prouvent aussi que l'abeille peut reconnaître son chemin au-delà de $3 \mathrm{~km}$, zone qu'elle prospecte pourtant rarement.

\section{RÉTENTION MNÉMONIQUE DE L'EMPLACEMENT DU TROU DE VOL}

La mémoire de l'abeille lui est aussi d'un grand secours pour retrouver l'emplacement exact de la ruche dans un rayon de quelques mètres, et l'emplacement du trou de vol. I,ECOMTE observe par exemple quand la ruche a subi une simple rotation de $I 80^{\circ}$ sur place, que les abeilles continuent à se diriger vers l'ancien emplacement de ce trou de vol, trois semaines au moins après le retournement. Or, cette période de trois semaines correspond à la durée de vie d'une abeille en tant que butineuse. Après ce laps de temps, les abeilles rentrent directement à la ruche, mais ce sont d'autres onorières, qui ont appris lors de leurs premières sorties à s'orienter par rapport à la nouvelle position du trou de vol. D'ailleurs, au cours des jours qui suivent le retournement, le nombre des butineuses qui se dirigent vers l'ancien trou de vol va en s'amenuisant, sans doute à cause de la mortalité progressive des vieilles butineuses.

A la suite d'une longue période de gel, en février I956, j'ai été surpris de constater qu'après 30 jours de claustration due au fait que la température ne permettait pas les sorties, les abeilles avaient gardé une mémoire exacte de la situation du trou de vol. En effet, nous avions besoin à la Station de Recherches Apicoles de jeunes larves en permanence ; et nous 
forcions la ponte des reines en plaçant les ruches dans un local chauffé, maintenu à température constante $\left(20-22^{\circ}\right)$. Le trou de vol de chaque ruche communiquait avec l'extérieur par un tuyau de $14 \mathrm{~cm}$ de diamètre, long de I m environ. Au cours des visites de surveillance, qui nécessitaient l'ouverture de la ruche, les abeilles se répandaient en plus ou moins grand nombre dans la pièce chauffée, et sortaient par une petite fenêtre, qu'on fermait aussitôt. Filles ne pouvaient alors revenir à la ruche que par le trou de vol extérieur, là où débouchait le tuyau. (fig. $\mathrm{n}^{0} \mathrm{I}$ ). Au cours de la sortie par la fenêtre, elles ne pouvaient pas repérer l'emplacement du trou de vol comme elles le font d'ordinaire, mais seulement se souvenir d'une manœuvre compliquée qu'elles avaient apprise un mois auparavant. Ajoutons que plusieurs tuyaux partant de différentes ruches
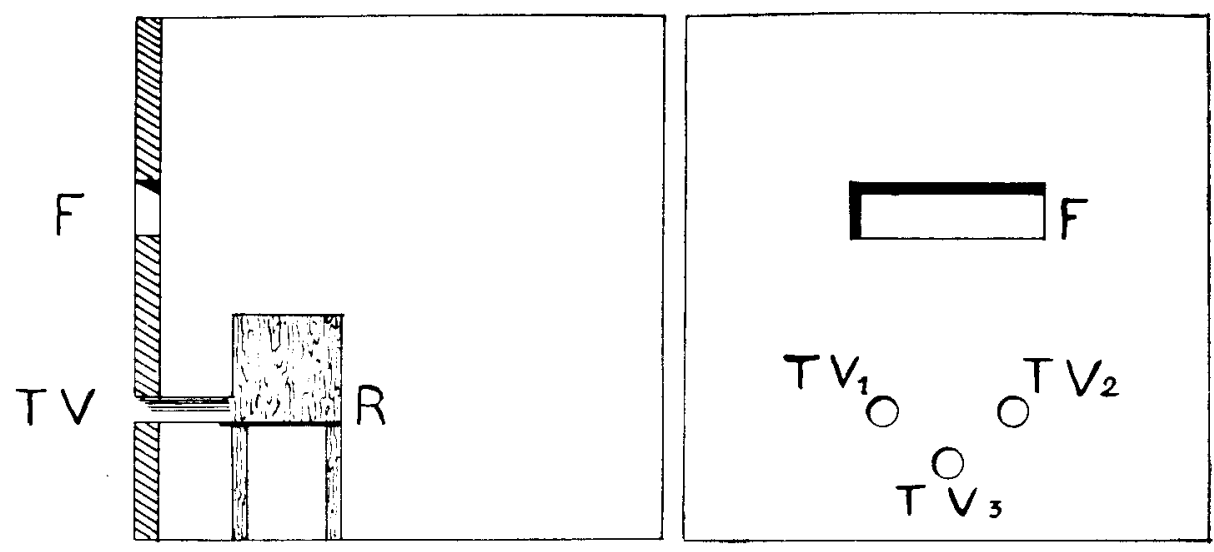

FiG. I. - A gauche : coupe du local chauffé avec à l'intérieur une ruche (R). TV, trou de vol. F, Fenêtre.

$\Lambda$ droite : vue de dehors montrant la position des 3 trous de vol $\left(T_{1}, T_{2}, T_{3}\right)$ et la fenétre $(F)$.

débouchaient côte à côte à l'extérieur, et ne se distinguaient par aucun repère spécial. Néanmoins, les abeilles regagnèrent leur ruche sans hésitation.

Un de mes collègues, $\mathrm{L}_{\mathrm{AVIE}}$ a observé un phénomène identique. Des abeilles conservées pendant un mois au frigidaire, au mois de juillet, avaient résisté au froid en formant une grappe très compacte. On les remit dehors à un emplacement différent de l'initial : or, elles rejoignirent ce dernier aussitôt. Ainsi donc, la rétention mémonique peut atteindre chez l'abeille un mois au minimum.

BUTLER (I949) prétend, à la suite d'observations faites sur des pourvoyeuses d'eau, que les abeilles d'hiver gardent la mémoire des lieux, depuis l'automne jusqu'aux premières sorties printanières, pratiquement donc pendant toute leur vie. Ceci confirme les résultats de LECOMTE sur les abeilles d'été. 


\section{Expériences sur la ménoire de l'emplacement.}

Nous avons entrepris une série d'expériences relatives à la mémoire de l'emplacement de la ruche par les abeilles sur une ruche isolée placée dans une prairie homogène d'herbes courtes. Cette prairie, située dans une large vallée, non loin de la Station de Recherches Apicoles, était bordée au Sud par un rideau d'arbres à 60 mètres de la ruche; au Nord, par des terres cultivées à IOO $\mathrm{m}$; à l'Ouest par des maisons à $300 \mathrm{~m}$; à 1'Hist, par un rideau d'arbres à $600 \mathrm{~m}$.

a) Une ruche est apportée d'un rucher situé à $30 \mathrm{~km}$. : les abeilles ignorent donc tout de leur nouvel emplacement. I,e trou de vol est dirigé vers le sud. 24 heures après, nous déplaçons cette ruche de $5 \mathrm{~m}$ en arrière, en laissant le trou de vol orienté du même côté (le transfert s'effectue vers I 4 heures au moment de la pleine activité des butineuses). Or, ces 24 heures ont suffi aux abeilles pour repérer exactement la position de la ruche. De retour du butinage, elles tourbillonment aussitôt près de l'ancien emplacement, totalement incapables de rentrer clans la ruche qui se trouve pourtant seule et bien visible au milieu de la prairie aux herbes courtes. Certaines abeilles volent en cercle sans arrêt; d'autres se posent près de l'ancien emplacement, attirées peut-être par une rémanence odorante, ou bien attirées par l'emplacement lui-même, qu'elles ne peuvent localiser pourtant qu'à l'aide de repères éloignés de $60 \mathrm{~m}$ au minimum. Cet affolement, ces tourbillons dans un rayon de $5 \mathrm{~m}$ cessent si Io minutes après le transfert, nous rapportons la ruche à sa place initiale. Les abeilles rentrent alors en masse et tout revient très vite dans l'ordre.

b) Dès que le calme est revenu, nous déplaçons à nouveau la ruche de la même distance et dans la même direction. Nouvelle effervescence, semblable à la première. Nous sommes restés nous-mêmes à I m de 1'ancien emplacement, et nous constatons que beaucoup d'abeilles s'agrippent à nous. Filles ne rentreront à leur ruche que si, au cours du quart d'heure suivant, nous la rapportons à l'emplacement initial. Cette expérience prouve avec quelle précision les abeilles repèrent leur ruche, mais aussi, que la vision de celle-ci ne leur sert qu'à très courte distance : elles ne l'aperçoivent pas, bien qu'elles ne se trouvent qu'à 5 mètres ; mais pourquoi l'observateur situé à I mètre joue-t-il alors le rôle de perchoir pour les butineuses affolées?

c) Deux jours après son installation dans la prairie, nous éloignons la même ruche d'I $m$ seulement en arrière (transfert toujours opéré en période de grande activité). Alors les abeilles très agitées tournent dans un demi cercle de rayon très court $(40 \mathrm{~cm})$ en avant du trou de vol; leur densité diminue rapidement lorsqu'on s'éloigne de la ruche et à I $\mathrm{m}$ on n'en voit plus que quelques unes qui tourbillonnent à peu près 
isolées. (fig. $n^{\circ}$ 2). Au retour, les butineuses se dirigent directement vers l'ancien emplacement (A) de la ruche et du trou de vol, qu'on retrouve facilement grâce à l'amas d'abeilles chargées de pollen qui s'y agglomèrent.

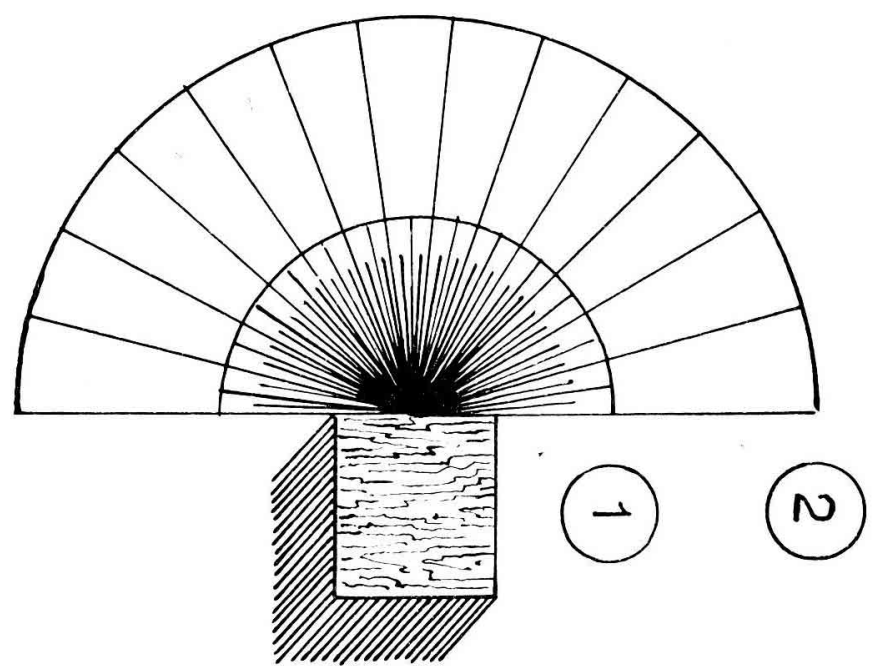

Fig. 2. - Vue de dessus d'une ruche : l'intensité des hachures est proportionnelle à lactivité des abeilles devant le trou de vol de la ruche.

Mais on ne voit plus l'affolement signalé dans le cas du recul de $5 \mathrm{~m}$ et les abeilles arrivées en $A$ gagnent directement $B$ (nouvel emplacement). Ici, le stimulus visuel pourrait jouer. Le changement brusque de direction en $\mathrm{A}$ persistera 35 minutes après le transfert; mais aucune abeille ne rôde en arrière de la ruche.

d) A ce moment, déplaçons la ruche de 2 mètres en avant, soit I mètre en avant de $\mathrm{A}$ (emplacement $\mathrm{C}$ ) (fig. 3). Les butineuses qui revien-
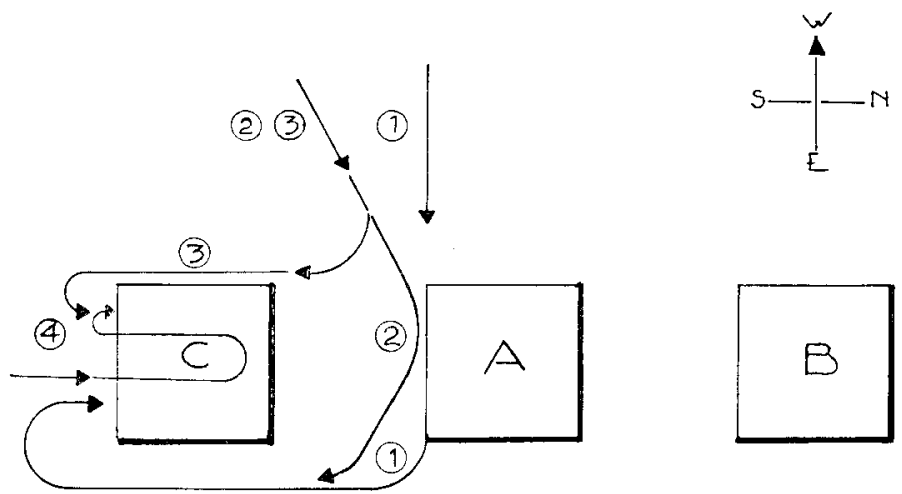

FIG. 3. - Vue de dessus de la ruche: A, ancien emplacement; B. ou C, nouveaux emplacements en arrière ( $\mathrm{I} \mathrm{m}$ ) ou en avant $(\mathrm{I} \mathrm{m})$ de l'emplacement initial. Le trou de vol se trouve à gauche sur la figure. Les flèches indiquent le trajet effectué par les abeilles rentrant à la ruche. Voir explications dans le texte page $163-564$. 


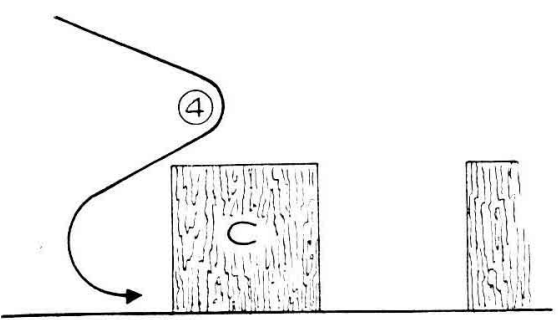

FIG. 3 bis. - Vue latérale d'ane ruche avancée d'un mètre par rapport à l'emplacement ini. tial. La flèche 4 reportée sur la figure 3 correspond au même trajet vu de dessus et. vu de profil.
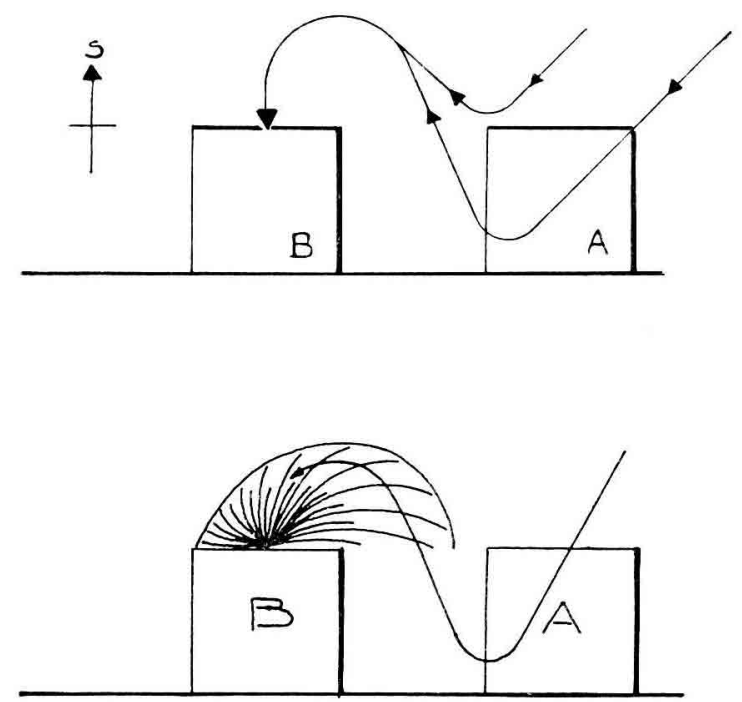

FIG. 4.

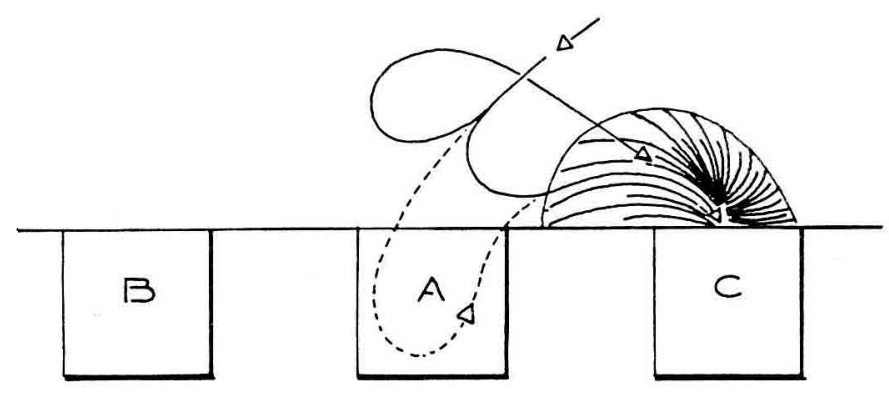

FIG. 5 .

Frg. 4-5. - Vue de dessus d'une ruche déplacée latéralement et flèches indiquant les trajets suivis par les abeilles. Comme pour la figure 2 , nous avons représenté par des hachures la répartition des abeilles devant la ruche après un déplacement d'un mètre à droite ou à gauche. 
nent effectuent alors des trajets variant avec la direction d'arrivée : celles qui viennent du Sud se dirigent vers l'ancien emplacement, puis, arrivées au-dessus de la ruche, virent de $180^{\circ}$ et gagnent directement la ruche (4). (fig. 3 et 3 bis). Les abeilles qui arrivent latéralement (I) gagnent directement 1'ancien emplacement puis en rasant le sol, contournent la ruche pour aborder le trou de vol; lorsqu'elles arrivent à $45^{\circ}(3-2)$ elles utilisent deux voies d'accès : ou bien elles contournent la ruche par derrière ou elles gagnent le trou de vol après avoir fait un coude. Si nous déplaçons le toit seul de la ruche pour le remettre en $A$, dans l'ancien emplacement, les abeilles tourbillonnent autour du toit avant de regagner la ruche. Le toit peut donc constituer un repère aussi important que les différentes marques colorées employées parfois sur la façade de la ruche, dans le but de la faire reconnaitre plus facilement par les abeilles.

e) Un déplacement d'un mètre vers la gauche (Est) provoque aussi une légère perturbation, car la plupart des butineuses viennent de la direction Sud-Ouest; elles se dirigent alors vers l'ancien trou de vol, puis apercevant la ruche à proximité, font un angle et gagnent, en rasant le sol, le nouvel emplacement. Le demi-cercle plein d'abeilles agitées déborde nettement sur le côté vers l'ancien emplacement (fig. 4).

f) Quinze minutes après, déplaçons la ruche de 2 mètres vers l'Ouest (position C), le demi-cercle d'abeilles agitées est déplacé aussi mais du côté de l'ancien emplacement, c'est-à-dire du côté opposé par rapport à l'expérience précédente. Même phénomène si la ruche est déplacée de 2 mètres et non plus de un (fig. 5).

g) Si alors, ayant déplacé la ruche de 5 mètres vers l'avant ou vers l'arrière, nous la laissons en repos à son nouvel emplacement, les perturbations déjà signalées continuent ; elles s'amplifient encore si le bond est de ro mètres et non plus de 5. Les abeilles recherchant la ruche tournent autour de l'emplacement, puis, comme l'ont vu beaucoup d'auteurs, augmentent le rayon de leur vol en montant de plus en plus, jusqu'au moment où certaines abeilles ont retrouvé la ruche et commencent à battre le rappel d'une manière très intense ; alors les rentrées débutent et s'accélèrent rapidement.

\section{LA RÉTENTION DURABLE}

Au bout de plusieurs jours, les abeilles continuent à venir à l'ancien emplacement, puis regagnent sans hésitation, en rasant le sol, leur ruche qui est éloignée de $5 \mathrm{~m}$. Le trajet suit alors une courbe représentée sur les figures ; tout le long de ce parcours les abeilles s'accrochent passivement à l'observateur s'il se place entre l'ancien et le nouvel emplacement. 
Lorsqu'on place en A une ruche vide, les butineuses de retour pénètrent dans cette ruche mais n'y demeurent pas, ne trouvant ni reine ni couvain qui puissent les y fixer; mais toutefois elles y pénètrent avec persistance et ce fait prouve qu'elles se souviennent que leur ruche autrefois était placée en cet endroit. Comme dans les expériences de $\mathrm{I}_{4}$ COMTE, le nombre des butineuses qui entre en A diminue progressivement, le nombre de celles qui rentrent directement en B augmente progressivement; il est bien probable que parmi elles, se trouvent les jeunes abeilles qui n'ont jamais connu l'emplacement A. On pourrait supposer aussi que les butineuses les plus jeunes, n'ont mémorisé l'emplacement A que depuis
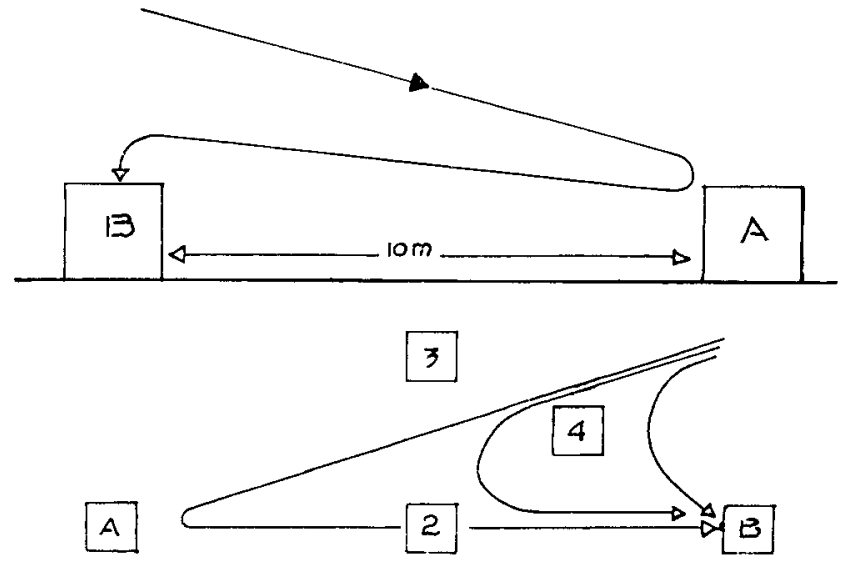

FIG. 6. - 'Transfert d'une ruche de A en B à une distance de $10 \mathrm{~m}$. Nous avons placé une ruche vide alternativement en $\mathrm{I}, 2,3,4$, entre l'ancien te le nouvel emplacement.

moins longtemps que les plus anciennes et l'abandonnent plus vite. Quoi qu'il en soit nous retrouvons, douze jours après la transposition, des abeilles qui effectuent encore ce parcours complexe.

Au cours de cette expérience, nous avons observé quelques abeilles rentrant de la direction $\mathrm{N}-\mathrm{O}$, c'est-à-dire qu'elles arrivaient à $45^{\circ}$ par derrière la ruche; elles contournaient toujours celle-ci dans le même sens pour regagner le trou de vol.

Après la transposition, nous avons placé alors une ruche vide à différents emplacements (fig. 6) : en (I) la ruche vide ne joue aucun rôle; en (2) de nombreuses abeilles retournent au-dessus de la ruche, à $30 \mathrm{~cm} \mathrm{du}$ toit pour quitter la piste normale et regagner la ruche B. En (3) quelques abeilles réagissent de façon analogue et quittent également leur piste normale pour rejoindre B. En (4) toutes les abeilles se dirigent vers B, 
mais après avoir montré une perturbation très nette. Les abeilles auraient donc gardé le souvenir de l'emplacement $\mathrm{A}$ de la ruche, mais aussi le souvenir de $A$ avec la ruche. La différence avec la première partie de l'expérience est la suivante : seule, ici, une certaine proportion d'abeilles est intéressée par l'ancien emplacement et le reste gagne B directement ; alors qu'au cours de la I ${ }^{\text {ere }}$ expérience, toutes les abeilles se dirigeaient vers $A$.

Eñ même temps que nous entreprenions ces recherches, FREE (I958) en exécutait dans le même sens en Angleterre. Bien que les techniques soient sensiblement différentes, les résultats concordent avec ceux de nos expériences.

\section{LES DEUX SORTES D'APPRENTISSAGE DE L'EMPLACEMENT}

Si l'on déplace une ruche venant de Io km, 2 heures après sa mise en place, les abeilles abandonnent aussitôt l'emplacement où elles n'ont demeuré que deux heures. Mais si la ruche est restée ro heures en place avant la transposition, les abeilles reviennent alors avec persistance à l'emplacement initial. Il existe donc en plus de la fixation rapide et photographique de l'emplacement de la ruche, que tous les auteurs ont signalée, une fixation qui demande plus de temps, mais qui semble produire des résultats à peu près irréversibles (les abeilles revenant à l'ancien emplacement pendant toute leur vie de butineuse).

\section{L'AMNÉSIE SUBITE DE L'ESSAIM}

Installé dès sa sortie dans une ruche vide à proximité de la ruche mère, l'essaim n'y retourne pas pour autant. I1 semble oublier totalement l'emplacement de la ruche dont il est sorti et, après le rite du vol de reconnaissance, chaque abeille parait adopter d'emblée la nouvelle position. Mais il suffit d'éliminer la reine de l'essaim, peu après son installation, pour que les émigrantes regagnent la souche : il n'y avait donc pas oubli, mais conflit de deux influences. Ia reine ne constitue pas la seule raison de la fixation, puisqu'il suffit de déplacer une ruche quelconque dans un rucher pour qu'elle perde toutes ses butineuses qui se réfugient au hasard dans les colonies les plus proches de l'ancien emplacement. C'est la fièvre d'essaimage, plus la présence de la reine, qui font adopter le nouveau gîte sans esprit de retour.

\section{DISCUSSION}

Si de très nombreux auteurs (résumé dans THORPE, I943) ont remarqué l'effet de la transposition des ruches sur le comportement des abeilles, 
il ne semble pas qu'ils se soient attachés à mesurer la ténacité de la rétention. D'autre part, HERTZ a signalé (oralement) à THORPE les difficultés qu'on éprouve à dresser des abeilles à venir récolter du sirop sur un coin de table : à ce stade, le repérage semble visuel et les abeilles sont facilement dérangées par le moindre changement dans l'environnement mais plus tard, des facteurs kinesthésiques semblent l'emporter de plus en plus et l'abeille devient moins sensible aux facteurs visuels. Nous trouvons ici un parallélisme exact avec nos expériences : lorsque la ruche vient d'être transportée dans un emplacement entièrement nouveau, il semble que dans les deux heures qui suivent la transposition, les abeilles se guident surtout d'après des repères visuels, et qu'un petit déplacement d'un mètre ou plus soit facilement corrigé. Ce n'est qu'au bout d'une dizaine d'heures que le repérage, dans la zone d'arrivée, devient de plus en plus kinesthésique et stéréotypé et qu'apparaît 1'attachement durable à l'emplacement. WoLf' a montré aussi que c'étaient les abeilles les plus âgées qui persistaient le plus à retourner dans l'ancien emplacement. Nous montrons quant à nous, qu'elles y retournent toute leur vie et que la fixation une fois faite semble irréversible. Notons qu'il n'en est pas de même chez Vespa Vulgaris qui retrouve facilement son nid déplacé de I à 6 mètres et dont le comportement à cet égard est bien plus plastique que celui de l'abeille (Husing).

\section{RÉSUMÉ}

$I^{0}$ Les théories qui n'admettent chez l'abeille qu'une brève capacité de rétention, limitée à trois ou quatre jours, sont controuvées par nos expériences ; nous avons démontré qu'une rétention de l'ancien emplacement, manifestée par les inflexions du parcours de retour à cet endroit, persiste pendant au moins douze jours. Chez les abeilles soumises artificiellement au froid ou en plein hiver, la rétention se manifeste pendant un mois au minimum.

$2^{\circ}$ Dans la localisation de la ruche à brève distance ( $\mathrm{I}$ à $2 \mathrm{~m}$ ) les stimuli visuels jouent certainement un rôle. Mais l'ancien emplacement dépourvu de toute marque visible est cependant bien reconnu et le parcours s'infléchit pendant très longtemps au-dessus de lui. Cela provient sans doute de la rétention des repères lointains, permettant, en dehors de tout repère proche, une orientation bien plus précise qu'on n'aurait pu le supposer.

$3^{\circ}$ Mais il faut distinguer entre : a) l'orientation quasi instantanée relevant de "l'insight " ou de l'apprentissage latent (THORPE) qui correspond sans doute aur vol stationnaire devant la ruche et se manifeste aussitôt dès que la ruche est placée dans un site entièrement nouveau, et b) " l'habituation " à l'emplacement, exigeant un maintien de plusieurs 
heures au même endroit et des parcours répétés. Seules les abeilles placées dans la situation (b) montrent une mémoire durable après déplacement de la ruche. Il paraît probable qu'au-delà d'une certaine marge d'habituation, la fixation de l'emplacement de la ruche est définitive et dure jusqu'à la mort de la butineuse.

$4^{\circ}$ I'abeille est sujette à des périodes d'anamnèse au cours desquelles 1'ancien emplacement paraît définitivement oublié. Il en est ainsi au cours de l'essaimage : l'essaim peut se fixer sans difficulté à quelques mètres de la souche. Le même résultat peut être obtenu en greffant des cupules de cire munies d'une jeune larve à l'intérieur d'un groupe d'abeilles orphelin. Mais si on enlève les cupules royales ou la reine, alors les abeilles retournent à la souche.

$5^{\circ}$ I'oubli n'était done qu'apparent et un facteur tout autre (reine ou élevage royal) inhibait seulement le retour au premier gite. Mais la reine seule ne suffit pas à provoquer cette inhibition; il y faut des périodes de crise comme l'essaimage ; autrement, il suffit de déplacer la ruche dans un rucher pour que toutes ses butineuses l'abandonnent et se réfugient dans les colonies les plus voisines de 1'ancien emplacement.

\section{SUMMARY}

I) Bees are able to remember the location of their hive during a long time : I 2 days in a normal period of activity and 30 days if bees are placed at $0-4^{\circ} \mathrm{C}$.

2) For the localisation of the hive at short distance ( $\mathrm{I}-2 \mathrm{~m}$ ) visual stimuli are important. But the ancient place even without visual marks is however well memorized. One can observe in the return flight of bees a deviation towards it during a long time. Perhaps important distant marks remain memorized a longer time than supposed in previous studies.

3) But we must distinguish between : a) (instantaneous orientation, which is manifest when a hive is placed in a new site ;

b) the "habituation " at the location which demands staying during several hours at the same place. Only the bees in condition $(b)$ show durable memory after hive translation. Perhaps, after some " habituation " location-engram becomes irreversible till the death of bees.

4) Bees show "anamnesis " periods during which they definitevely forget the ancient site : in period of swarming for instance, bees can be moved to a short distance. The same anamnesis can be seen among queenless bees provided with royal cells or young larvae, but if royal cells or larvae are taken off, the bees return to the ancient location.

5) Forgetting was only apparent. The presence of the queen is not sufficient to provoke the anamnesis, except during periods of swarming : after removing a hive in a apiary, all foragers abandon it and join the other hives, near the ancient site. 


\section{RÉFÉRENCES BIBLIOGRAPHIQUES}

Buther (C. G.). - The honeybee. Clarendon Press, Oxford, 1949.

Chauvin (R.). - Etudes sur le comportement de Bl. atella germanica dans divers types de labyrinthes. Bull. Biol. Fr. Belg., LXXI, 93-I28, I947.

FREE (J. B.). - The ability of worker honeybees (Apis mellifera) to learn a change in the location of their hives. Animal behaviour. Vol. VI, $\mathrm{n}^{\circ} 3-4$, July-oct., 1958 .

Von Frisch (K.). - Der Farbensinn und Formensinn der Biene. Zool. Jahrb. Allg. Zool., 35, I-I88, I9I4.

VoN FrIsch (K.), IINDAUER (M.). - Himmel und Erde in Konkurrenz bei der Orientierung der Bienen. Naturwiss, II, 245-53, I954.

LECOMTE (J.). - Formation de pistes par les butineuses d'une ruche ayant subi une rotation de $180^{\circ} . Z$. Bienenforsch., 3, I28-33, I956.

Husing (J. O.). - Beobachtungen über die Orientierung am Nest bei Vespa Vulgaris. Abh. Ber. Naturk., IX, I27-50, I955.

Thorpe (W. H.). - Types of learning in insects and other Arthropods. British J. Exper. Psychol., XXXIV, 20-3I, I943.

Wolf (E.). - Uber das Heimkehrvermogen der Bienen. Z. iergl. Physiol., 3, H. 6, 1926 . 